\title{
Six-bilayer periodic structures in GaN grown on GaAs(001)
}

\author{
$\operatorname{AUTHOR}(S)$ :
}

Funato, M; Ishido, T; Fujita, S; Fujita, S

\section{CITATION:}

Funato, M ...[et al]. Six-bilayer periodic structures in GaN grown on GaAs(001). APPLIED PHYSICS LETTERS 2000, 76(3): 330-332

\section{ISSUE DATE:}

2000-01-17

URL:

http://hdl.handle.net/2433/39634

\section{RIGHT:}

Copyright 2000 American Institute of Physics. This article may be downloaded for personal use only. Any other use requires prior permission of the author and the American Institute of Physics. 


\title{
Six-bilayer periodic structures in GaN grown on GaAs(001)
}

\author{
Mitsuru Funato, Teruki Ishido, Shizuo Fujita, and Shigeo Fujita \\ Department of Electronic Science and Engineering, Kyoto University, Kyoto 606-8501, Japan
}

(Received 4 May 1999; accepted for publication 15 November 1999)

\begin{abstract}
We have observed six-bilayer periodic structures in $\mathrm{GaN}$ grown on $\mathrm{GaAs}(001)$. The periodicity occurs along the zinc-blende(ZB)-[111]A direction, suggesting that it originates from stacking faults on close-packed planes. GaN grown on GaAs includes both ZB and wurtzite phases as a result of formation of stacking faults and the periodic structures are mostly located between these two crystalline phases. Based on this observation, possible layer stacking sequences are proposed, which are classified as 6H polytypes. (C) 2000 American Institute of Physics. [S0003-6951(00)00303-X]
\end{abstract}

Zinc-blende (ZB) and wurtzite (W) are the most common polytypes of binary octet semiconductors. ${ }^{1-3}$ The stable structure of $\mathrm{GaN}$ is wurtzite and devices including the light emitting diodes now available commercially have been fabricated with $\mathrm{W}-\mathrm{GaN}^{4}{ }^{4,5}$ On the other hand, by selecting proper substrates and growth conditions, the metastable ZB structure can also be grown epitaxially. ${ }^{6-11}$ For example, there are many reports on the successful growth of $\mathrm{ZB}-\mathrm{GaN}$ on cubic (001) substrates such as $\mathrm{GaAs}^{6-8} \mathrm{Si}^{6}{ }^{9}$ and $3 \mathrm{C}-\mathrm{SiC},{ }^{10,11}$ although inclusion of the $\mathrm{W}$ phase has not been completely eliminated. The polytypes so far found in $\mathrm{GaN}$ are $\mathrm{W}$ and $\mathrm{ZB}$, and other structures such as $4 \mathrm{H}$ and $6 \mathrm{H}$ have not yet been observed. As is well known, however, some semiconductors such as $\mathrm{ZnS}$ and $\mathrm{SiC}$ exhibit surprisingly many (more than 200) polytypes, ${ }^{12,13}$ depending on the growth parameters. This motivates us to investigate $\mathrm{GaN}$ in terms of polytypism. For this purpose, a theoretical study by Wright ${ }^{14}$ provides an interesting clue; $\mathrm{W}$ is the most stable structure in the case of $\mathrm{GaN}$, and this makes it energetically favorable to introduce stacking faults in ZB films because $\mathrm{ZB}$ faults on close-packed planes inevitably contain the lower-energy W stacking. Indeed, as long as we observed by transmission electron microscopy (TEM), $\mathrm{ZB}-\mathrm{GaN}$ grown on $\mathrm{GaAs}(001)$ included a much greater number of stacking faults than $\mathrm{W}-\mathrm{GaN}$ grown on $\mathrm{Al}_{2} \mathrm{O}_{3}(0001)$ did. It must be noted, here, that hexagonal polytypes consist of the mixture of the $\mathrm{ZB}$ and $\mathrm{W}$ stacking and, consequently, are more stable than the pure ZB structure. Therefore, it is reasonably assumed that various polytypes are formed in $\mathrm{GaN}$ layers grown on cubic substrates as a result of generation of stacking faults. In this study, we investigate $\mathrm{GaN}$ grown on $\operatorname{GaAs}(001)$ structurally and demonstrate the presence of polytypes other than ZB and $\mathrm{W}$.

A GaN thin film was grown on a GaAs(001) substrate by atmospheric-pressure metalorganic vapor phase epitaxy (MOVPE). The gallium and the nitrogen source precursors were triethylgallium (TEGa) and dimethylhydrazine (DMHy), respectively. The GaAs substrate was etched in a solution of $\mathrm{H}_{2} \mathrm{SO}_{4}: \mathrm{H}_{2} \mathrm{O}_{2}: \mathrm{H}_{2} \mathrm{O}=5: 1: 1$ at $40^{\circ} \mathrm{C}$ for $1 \mathrm{~min}$. In order to remove the surface oxide, the GaAs substrate was thermally annealed at $600{ }^{\circ} \mathrm{C}$ for $10 \mathrm{~min}$ in a hydrogen ambient. Nitridation of the GaAs surface was not conducted. A $\mathrm{GaN}$ buffer layer about $20 \mathrm{~nm}$ thick was grown on $\mathrm{GaAs}$ at $600^{\circ} \mathrm{C}$ under a V/III ratio of 100 . Then a GaN layer was grown at a higher temperature of $795{ }^{\circ} \mathrm{C}$ with a V/III ratio of 50. Under those conditions, the growth rate was about 0.35 $\mu \mathrm{m} / \mathrm{h}$ for both the low and high temperature grown layers.

From a crystallographic point of view, polytypism is a phenomenon whereby a compound exhibits a variety of periodic layered structures along the $\mathrm{ZB}-\langle 111\rangle$ direction, which is equivalent to the hexagonal $\langle 0002\rangle$ direction ( $c$ axis). For example, $\mathrm{ZB}(3 \mathrm{C})$ is characterized by an $A B C A B C \cdots$ sequence, whereas $\mathrm{W}(2 \mathrm{H})$ by an $A B A B A B \cdots$ sequence. In those notations, each letter stands for an ordered pair of cation and anion layers. Thus, in order to investigate polytypes in $\mathrm{GaN}$ on $\mathrm{GaAs}(001),(110)$ and $(\overline{1} 10)$ cross sections were observed by TEM operated at $200 \mathrm{kV}$. The indices of the cross sections were identified by carefully preparing the specimens so as not to lose the already-known crystalline orientation of the substrates. The electron diffraction (ED) pattern obtained from the (110) cross section, which includes information on stacking features of (111)B planes, shows only the fundamental diffraction spots of the ZB phase. On the other hand, in the ED pattern from the (110) cross section, many additional spots were observed. Therefore, discussion hereafter will be concentrated on the (110) cross section, that is, on the (111)A layer stacking.

In the bright field image shown in Fig. 1(a), stacking faults inclined in the (111)A planes can be seen. The microbeam ED patterns were observed at several regions labeled (1)-(3) in the schematic view [Fig. 1(b)] and are compared
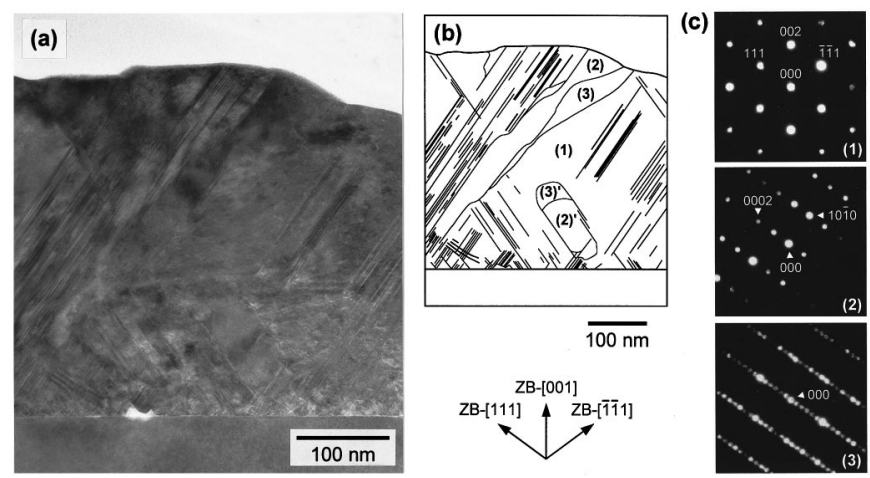

FIG. 1. (a) TEM bright field image of the (110) cross section of GaN grown on $\operatorname{GaAs}(001)$ and (b) its schematic view. The ED patterns shown in (c) were measured at the regions (1)-(3) in the schematic view, which indicate the presence of pure $\mathrm{ZB}$, pure $\mathrm{W}$, and a six-bilayer periodic structure, respectively. 

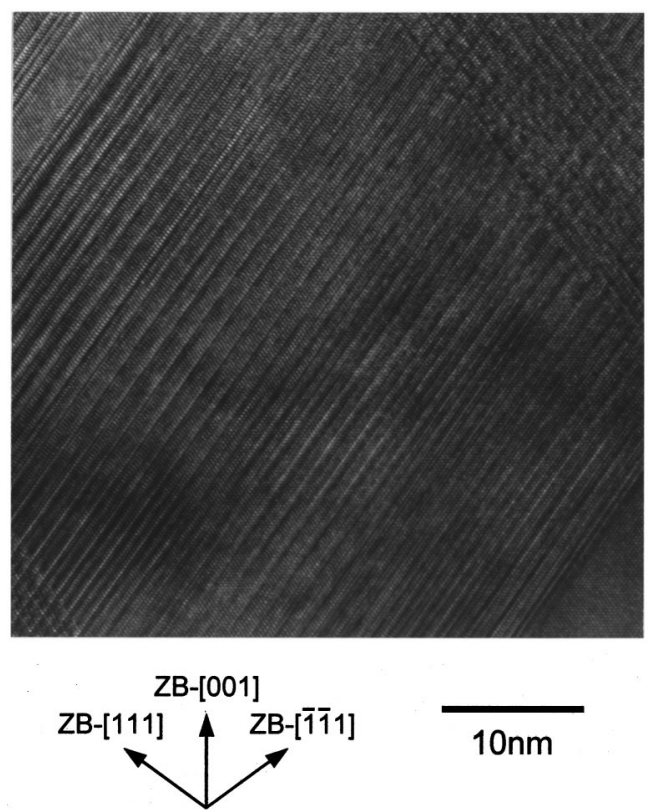

\section{$10 \mathrm{~nm}$}

FIG. 2. High-resolution image of the region including the six-bilayer periodicity.

in Fig. 1(c). The ED patterns from the regions (1) and (2) prove that these regions consist of the pure $\mathrm{ZB}$ and $\mathrm{W}$ phase, respectively. It was confirmed that the $\mathrm{ZB}-[111]$ direction was parallel to the $\mathrm{W}$-[0002] direction ( $c$ axis). On the other hand, the ED pattern from the region (3) shows satellite spots aligned in the ZB-[111] direction. The distance between the neighboring satellite spots is exactly $1 / 6$ of that between 000 and ZB-111 diffraction spots. These findings indicate that the sample involves the periodic structure along the ZB-[111] direction with the periodicity of 6 times as thick as the ZB$\{111\}$ spacing. The ZB- $\{111\}$ spacing, which is equal to the $\mathrm{W}-\{0002\}$ spacing, is the bilayer (monomolecular) spacing in the $\langle 111\rangle$ direction, that is, $2.6 \AA$. Therefore, the period of the structure is estimated to be $15.6 \AA$. It should be noted that the period is almost always six bilayers, indicating that the closest-packing structure is preserved. In Fig. 1, other variants for the $\mathrm{W}$ phase and the periodic structure which orient the $\mathrm{ZB}-[\overline{\mathrm{1}} \overline{1} 1]$ direction are also found, for example, at the positions indicated as $(2)^{\prime}$ and $(3)^{\prime}$, respectively. In this study, regarding the periodic structures, the directions along which the periodicity occurs are denoted as the "c axis." The $c$ axis of the periodic structure is parallel to either the ZB-[111] or ZB-[1̄11] direction. The layer thickness of the periodic structures along their $c$ axis ranged from 50 to 100 $\mathrm{nm}$ and the fractional occupancy against the entire area of the cross section was estimated to be about $5 \%$, though these structural parameters depend on growth conditions.

In order to identify the layer stacking sequence of the structure with six-bilayer periodicity, a high-resolution (HR) image was observed at the corresponding region. The result is shown in Fig. 2. In this region, the $c$ axis of the periodic structure is parallel to the ZB-[111] direction and the periodicity spreads over $50 \mathrm{~nm}$ along the $c$ axis. The HR image again confirms the periodicity of $15.6 \AA$, but we could not determine the stacking sequence due to the diffused spots, which are probably caused by strain induced distribution of atom positions within the specimen thickness for the TEM Downloaded 30 May 2007 to 130.54.110.22. Redistribution subject

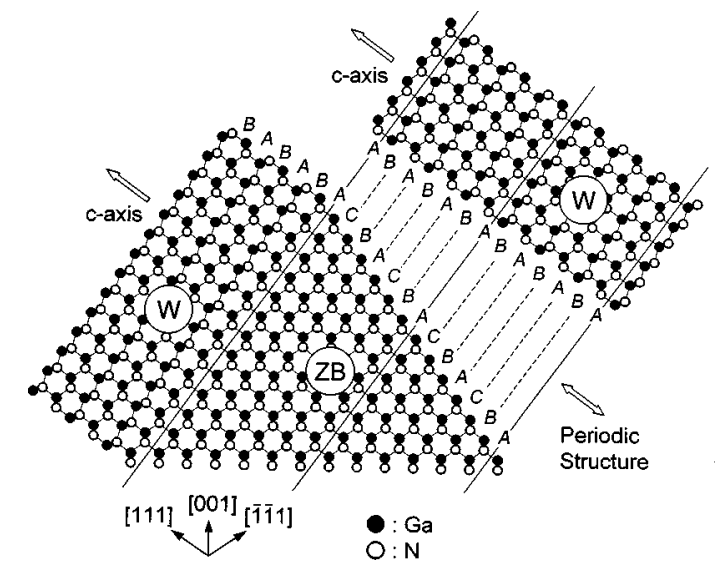

FIG. 3. Structural model for the formation of the periodic structure.

observation. The origin of this distribution is considered to be related to the formation mechanism of the six-bilayer periodic structures and will be discussed below in detail.

Let us consider plausible stacking sequences in the periodic structures. For this, it is critical to elucidate its origin. Referring to Fig. 1 again, it is found that the periodic structures are sandwiched between the $\mathrm{ZB}$ and $\mathrm{W}$ phases in the directions perpendicular to their $c$ axes; the region (3) is between (1) and (2), while (3)' is between (1) and (2)' . In other places, which are not shown in Fig. 1, the periodic structure was observed between two $\mathrm{W}$ variants with different orientations, that is, $c$ axis $\| \mathrm{ZB}-[111]$ and ZB-[1̄11]. However, this structure is essentially identical with the structures found in Fig. 1, in which the periodic structures are located between the ZB and $\mathrm{W}$ phases, because the ZB- $\{111\}$ planes are equivalent to the $\mathrm{W}-\{0002\}$ planes. Therefore, we discuss the sandwich structure with $\mathrm{ZB}$ and $\mathrm{W}$.

Figure 3 schematically illustrates this structural model. When the $\mathrm{ZB}$ and $\mathrm{W}$ phases are stacked along the $c$ axis of the $\mathrm{W}$ phase, these regions may be separated by a single stacking fault due to the equivalency of $\mathrm{ZB}-\{111\}$ and $\mathrm{W}-\{0002\}$ as shown on the left-hand side of Fig. 3. On the other hand, when the stacking direction of $\mathrm{ZB}$ and $\mathrm{W}$ is perpendicular to the $c$ axis as on the right-hand side of Fig. 3, spatial shifts of atom positions must occur. The layer stacking sequence along $\mathrm{ZB}-\langle 111\rangle$ for $\mathrm{ZB}$ is $A B C A B C \cdots$, while $A B A B A B \cdots$ for $\mathrm{W}$. Therefore, if $\mathrm{ZB}$ and $\mathrm{W}$ transform into each other directly, the difference of the latter four sequences ( $C A B C$ for $\mathrm{ZB}$ and $A B A B$ for $\mathrm{W}$ ) brings the shift of the atoms in the corresponding sequences in the vicinity of the $\mathrm{ZB}-\mathrm{W}$ interface, resulting in large strain. In order to avoid the introduction of the large strain, a transition region with the six-bilayer periodicity will be formed. The periodicity of six bilayer is derived from the complete agreement of the layer stacking sequences of $\mathrm{ZB}$ and $\mathrm{W}$ every six-bilayer stacking, as is deduced from the sequences of $\mathrm{ZB}$ $(A B C A B C \cdots)$ and $\mathrm{W}(A B A B A B \cdots)$, or from Fig. 3.

As for the stacking sequences in the periodic structures, the sequences which induce much more strain than the direct transformation between $\mathrm{ZB}$ and $\mathrm{W}$ may be ruled out. For example, an $A C B C B C \cdots$ sequence is different by four sequences from both $\mathrm{ZB}$ and $\mathrm{W}$, and therefore it will hardly appear. Furthermore, the experimental result that the closestpacking is maintained in the periodic structures as was o AlP license or copyright, see http://apl.aip.org/apl/copyright.jsp 
mention in the discussion with Fig. 1 allows us not to consider the stacking of the same sequence such as $A A$, $B B$, and $C C$. Taking these factors into account, it is found that possible sequences are $A B C B A C \cdots, A B C B A B \cdots$, and $A B A B A C \cdots$. The $A B C B A C \cdots$ sequence differs by two sequences from both $\mathrm{ZB}$ and $\mathrm{W}$. For the latter two, $A B C B A B \cdots$ and $A B A B A C \cdots$, on the other hand, the deviation from $\mathrm{ZB}$ is counted as three sequences, while one sequence from $\mathrm{W}$. Therefore, as the stacking sequences in the periodic structures, the transition of $\mathrm{ZB}(A B C A B C \cdots) \leftrightarrow A B C B A C \cdots \leftrightarrow(A B C B A B \cdots$ or $A B A B A C \cdots) \leftrightarrow \mathrm{W}(A B A B A B \cdots)$ is a plausible candidate. It should be noted that those sequences do not need to appear with the similar fractional occupancy and that either sequence can dominate the periodic structure. In the case of Fig. 2, these sequences would be mixed and, consequently, we could not identify the stacking sequence uniquely.

In the above discussion, the sandwich structure was used as a structural model. In the actual crystal growth, however, the growth direction of the GaN layer ([001]) is different from the direction in which the periodicity occurs (ZB[111]A). Therefore, it is not determined at the onset of the periodic structures on the $\mathrm{ZB}$ or $\mathrm{W}$ phase whether those are finally sandwiched by ZB and W. From this fact, the formation process of the six-bilayer structures is deduced as follows; when a driving force to be $\mathrm{W}$ is applied to the $\mathrm{ZB}$ phase, or vice versa, the periodic structure is formed and continues to grow unless it is terminated. An example of the driving force is atomically rough surfaces. Let us suppose that there is a hole which is surrounded with $\{111\}$ planes on the outermost plane of the $\mathrm{ZB}$ phase. If an adatom is captured at the hole and is going to occupy the $\mathrm{W}$ sublattice on the ZB-(111) plane, the relation between the embedded atom and the ZB-(1)1 ) plane realize the situation shown on the right-hand side of Fig. 3. Therefore, in this case, the periodic structure whose $c$ axis is in the ZB-[111] direction will be formed. The periodic structure along $\mathrm{ZB}-[\overline{1} \overline{1} 1]$ as well can appear via the same mechanism.
To summarize, the structural properties of GaN grown on GaAs(001) were investigated by TEM in terms of polytypism. It was revealed that there was the structure with sixbilayer periodicity along the ZB-[111]A direction, which is indicative of the presence of $6 \mathrm{H}$ polytypes. Its origin was attributed to the transformation of $\mathrm{ZB} \leftrightarrow \mathrm{W}$ along the direction perpendicular to the $c$ axis of the $\mathrm{W}$ phase. The present study demonstrates the possibility of the presence of many polytypes in $\mathrm{GaN}$ grown on $\mathrm{GaAs}(001)$.

The authors acknowledge Professor N. Kuwano at Kyusyu University for his suggestive discussion and Dr. K. Takahashi at the MST (Foundation for Promotion of Material Science and Technology of Japan) for his collaboration in the TEM studies.

${ }^{1}$ P. Villars and L. Calvert, Pearson's Handbook of Crystallographic Data for Intermetallic Phases (American Society for Metals, Metals Park, OH, 1985).

${ }^{2}$ R. W. G. Wyckoff, Crystal Structure, 2nd ed. (Interscience, New York, 1963), Vol. 1.

${ }^{3}$ E. Parthe, Crystal Chemistry of Tetrahedral Structures (Gordon and Breach, New York, 1964)

${ }^{4}$ S. Nakamura, M. Senoh, N. Iwasa, S. Nagahama, T. Yamada, and T. Mukai, Jpn. J. Appl. Phys., Part 2 34, L1332 (1995).

${ }^{5}$ S. Nakamura, M. Senoh, S. Nagahama, N. Iwasa, T. Yamada, T. Matsushita, H. Kiyoku, Y. Sugimoto, T. Kozaki, H. Umemoto, M. Sano, and K. Chocho, Appl. Phys. Lett. 72, 2014 (1998).

${ }^{6}$ S. Miyoshi, K. Onabe, N. Ohkouchi, H. Yaguchi, R. Ito, S. Fukatsu, and Y. Shiraki, J. Cryst. Growth 124, 439 (1992).

${ }^{7}$ A. Nakadaira and H. Tanaka, J. Electron. Mater. 26, 320 (1997).

${ }^{8}$ H. Tachibana, T. Ishido, M. Ogawa, M. Funato, Sz. Fujita, and Sg. Fujita, J. Cryst. Growth 196, 41 (1999).

${ }^{9}$ T. Lei, M. Fanciulli, R. J. Molnar, T. D. Moustakas, R. J. Graham, and J. Scanlon, Appl. Phys. Lett. 59, 944 (1991).

${ }^{10}$ M. J. Paisley, Z. Sitar, J. B. Posthill, and R. F. Davis, J. Vac. Sci. Technol. A 7, 701 (1989).

${ }^{11}$ H. Okumura, S. Misawa, T. Okahisa, and S. Yoshida, J. Cryst. Growth 136, 361 (1994).

${ }^{12}$ S. Mardix, Phys. Rev. B 33, 8677 (1986).

${ }^{13}$ Polymorphism and Polytypism in Crystals, edited by A. R. Verma and P. Krishna (Wiley, Inc. New York, 1966).

${ }^{14}$ A. F. Wright, J. Appl. Phys. 82, 5259 (1997). 